\title{
Structure of the Hydrated (1014) Surface of Rhodochrosite $\left(\mathrm{MnCO}_{3}\right)$
}

\author{
Young-Shin Jun, Sanjit K. Ghose, Thomas P. Trainor, \\ Peter J. Eng, and Scot T. Martin
}

Supporting Information:

10 Pages

1 Table

2 Figure

2 Appendices 


\begin{tabular}{|c|c|c|c|c|c|c|c|c|c|c|c|c|}
\hline \multirow{2}{*}{\begin{tabular}{|l} 
Layer \\
\end{tabular}} & & \multicolumn{4}{|c|}{ (A) Bulk termination } & \multicolumn{7}{|c|}{ (B) Relaxed surface without an oxygen overlayer } \\
\hline & & $\mathbf{x}$ & $\mathbf{y}$ & $\mathbf{z}$ & $\Sigma B V$ & $\mathbf{X}$ & $\mathbf{y}$ & $\mathbf{Z}$ & $\Delta \mathbf{z}[\AA]$ & $\mathbf{D W}\left[\AA^{2}\right]$ & Occ & $\overline{\Sigma B V}$ \\
\hline 1 & $\mathrm{O}_{1}(+)$ & 0.752 & -0.135 & 0.635 & 1.66 & $0.707(4)$ & $-0.14(1)$ & $0.697(5)$ & $0.35(3)$ & 0.57 & $0.83(4)$ & $1.43(3)$ \\
\hline 1 & $\mathrm{O}_{1}(+)$ & 0.252 & 1.135 & 0.635 & 1.66 & $0.207(4)$ & $1.14(1)$ & $0.697(5)$ & $0.35(3)$ & 0.57 & $0.83(4)$ & $1.43(3)$ \\
\hline 1 & $\mathrm{Mn}_{1}$ & 0.395 & 0.500 & 0.500 & 1.70 & $0.394(3)$ & $0.516(2)$ & $0.492(2)$ & $-0.05(1)$ & 0.51 & $0.85(4)$ & $1.56(3)$ \\
\hline 1 & $\mathrm{Mn}_{1}$ & 0.895 & 0.500 & 0.500 & 1.70 & $0.894(3)$ & $0.484(2)$ & $0.492(2)$ & $-0.05(1)$ & 0.51 & $0.85(4)$ & $1.56(3)$ \\
\hline 1 & $\mathrm{C}_{1}$ & 0.645 & 0.000 & 0.500 & 3.97 & $0.630(4)$ & $-0.01(1)$ & $0.523(5)$ & $0.13(3)$ & 0.48 & $0.83(4)$ & $3.97(3)$ \\
\hline 1 & $\mathrm{C}_{1}$ & 0.145 & 1.000 & 0.500 & 3.97 & $0.130(4)$ & $1.01(1)$ & $0.523(5)$ & $0.13(3)$ & 0.48 & $0.83(4)$ & $3.97(3)$ \\
\hline 1 & $\mathrm{O}_{1}$ & 0.645 & 0.270 & 0.500 & 2.00 & $0.630(4)$ & $0.26(1)$ & $0.510(5)$ & $0.06(3)$ & 0.57 & $0.83(4)$ & 1.94(3) \\
\hline 1 & $\mathrm{O}_{1}$ & 0.145 & 0.730 & 0.500 & 2.00 & $0.130(4)$ & $0.74(1)$ & $0.510(5)$ & $0.06(3)$ & 0.57 & $0.83(4)$ & $1.94(3)$ \\
\hline 1 & $\mathrm{O}_{1}(-)$ & 0.539 & -0.135 & 0.365 & 2.01 & $0.553(4)$ & $-0.16(1)$ & $0.363(5)$ & $-0.01(3)$ & 0.57 & $0.83(4)$ & $2.07(3)$ \\
\hline 1 & $\mathrm{O}_{1}(-)$ & 0.039 & 1.135 & 0.365 & 2.01 & $0.053(4)$ & $1.16(1)$ & $0.363(5)$ & $-0.01(3)$ & 0.57 & $0.83(4)$ & 2.07(3) \\
\hline 2 & $\mathrm{O}_{2}(+)$ & 0.857 & 0.635 & 0.135 & 2.00 & $0.848(4)$ & $0.635(4)$ & $0.143(4)$ & $0.05(2)$ & 0.57 & 1.00 & $2.00(3)$ \\
\hline 2 & $\mathrm{O}_{2}(+)$ & 0.357 & 0.365 & 0.135 & 2.00 & $0.348(4)$ & $0.365(4)$ & $0.143(4)$ & $0.05(2)$ & 0.57 & 1.00 & $2.00(3)$ \\
\hline 2 & $\mathrm{Mn}_{2}$ & 0.500 & 1.000 & 0.000 & 2.04 & $0.497(2)$ & $0.996(2)$ & $-0.003(2)$ & $-0.02(1)$ & 0.51 & 1.00 & $1.95(3)$ \\
\hline 2 & $\mathrm{Mn}_{2}$ & 0.000 & 0.000 & 0.000 & 2.04 & $-0.003(2)$ & $0.004(2)$ & -0.0 & $-0.02(1)$ & 0.51 & 1.00 & $1.95(3)$ \\
\hline 2 & $\mathrm{C}_{2}$ & 0.250 & 0.500 & 0.000 & 3.96 & $0.246(4)$ & $0.497(4)$ & $-0.001(4)$ & $-0.01(2)$ & 0.48 & 1.00 & $3.96(3)$ \\
\hline 2 & $\mathrm{C}_{2}$ & 0.750 & 0.500 & 0.000 & 3.96 & $0.746(4)$ & $0.503(4)$ & $-0.001(4)$ & $-0.01(2)$ & 0.48 & 1.00 & $3.96(3)$ \\
\hline 2 & $\mathrm{O}_{2}$ & 0.250 & 0.770 & 0.000 & 2.00 & $0.246(4)$ & $0.767(4)$ & $-0.006(4)$ & $-0.03(2)$ & 0.57 & 1.00 & $1.98(3)$ \\
\hline 2 & $\mathrm{O}_{2}$ & 0.750 & 0.230 & 0.000 & 2.00 & $0.746(4)$ & $0.233(4)$ & $-0.006(4)$ & $-0.03(2)$ & 0.57 & 1.00 & $1.98(3)$ \\
\hline 2 & $\mathrm{O}_{2}(-)$ & 0.143 & 0.365 & -0.135 & 2.00 & $0.145(4)$ & $0.358(4)$ & $-0.141(4)$ & $-0.03(2)$ & 0.57 & 1.00 & $2.04(3)$ \\
\hline 2 & $\mathrm{O}_{2}(-)$ & 0.643 & 0.635 & -0.135 & 2.00 & $0.645(4)$ & $0.642(4)$ & $-0.141(4)$ & $-0.03(2)$ & 0.57 & 1.00 & $2.04(3)$ \\
\hline \multicolumn{6}{|c|}{ (A) Bulk termination } & \multicolumn{7}{|c|}{ (C) Relaxed surface with an oxygen overlayer } \\
\hline $\mathrm{w}$ & $\mathrm{O}_{\mathrm{w}}$ & - & - & - & - & $0.91(1)$ & $0.36(1)$ & $0.941(9)$ & - & 2.04 & $0.84(10)$ & - \\
\hline $\mathrm{w}$ & $\mathrm{O}_{\mathrm{w}}$ & - & - & - & - & $0.41(1)$ & $0.65(1)$ & $0.941(9)$ & - & 2.04 & $0.84(10)$ & - \\
\hline 1 & $\mathrm{O}_{1}(+)$ & 0.752 & -0.135 & 0.635 & 1.66 & $0.76(1)$ & $-0.16(1)$ & $0.60(2)$ & $-0.20(1)$ & 0.57 & $0.94(5)$ & $1.80(3)$ \\
\hline 1 & $\mathrm{O}_{1}(+)$ & 0.252 & 1.135 & 0.635 & 1.66 & $0.26(1)$ & $1.16(1)$ & $0.60(2)$ & $-0.20(1)$ & 0.57 & $0.94(5)$ & $1.80(3)$ \\
\hline 1 & $\mathrm{Mn}_{1}$ & 0.395 & 0.500 & 0.500 & 1.70 & $0.390(3)$ & $0.514(2)$ & $0.499(2)$ & $0.00(1)$ & 0.51 & $0.84(4)$ & $1.92(3)$ \\
\hline 1 & $\mathrm{Mn}_{1}$ & 0.895 & 0.500 & 0.500 & 1.70 & $0.890(3)$ & $0.486(2)$ & $0.499(2)$ & $0.00(1)$ & 0.51 & $0.84(4)$ & $1.92(3)$ \\
\hline 1 & $\mathrm{C}_{1}$ & 0.645 & 0.000 & 0.500 & 3.97 & $0.646(5)$ & $-0.014(6)$ & $0.486(4)$ & $-0.08(2)$ & 0.48 & $0.94(5)$ & $3.97(3)$ \\
\hline 1 & $\mathrm{C}_{1}$ & 0.145 & 1.000 & 0.500 & 3.97 & $0.146(5)$ & $1.014(6)$ & $0.486(4)$ & $-0.08(2)$ & 0.48 & $0.94(5)$ & $3.97(3)$ \\
\hline 1 & $\mathrm{O}_{1}$ & 0.645 & 0.270 & 0.500 & 2.00 & $0.646(9)$ & $0.26(1)$ & $0.50(1)$ & $-0.02(7)$ & 0.57 & $0.94(5)$ & $1.95(3)$ \\
\hline 1 & $\mathrm{O}_{1}$ & 0.145 & 0.730 & 0.500 & 2.00 & $0.146(9)$ & $0.74(1)$ & $0.50(1)$ & $-0.02(7)$ & 0.57 & $0.94(5)$ & $1.95(3)$ \\
\hline 1 & $\mathrm{O}_{1}(-)$ & 0.539 & -0.135 & 0.365 & 2.01 & $0.53(1)$ & $-0.14(1)$ & $0.36(1)$ & $-0.05(6)$ & 0.57 & $0.94(5)$ & $2.11(3)$ \\
\hline 1 & $\mathrm{O}_{1}(-)$ & 0.039 & 1.135 & 0.365 & 2.01 & $0.03(1)$ & $1.14(1)$ & $0.36(1)$ & $-0.05(6)$ & 0.57 & $0.94(5)$ & $2.11(3)$ \\
\hline 2 & $\mathrm{O}_{2}(+)$ & 0.857 & 0.635 & 0.135 & 2.00 & $0.853(4)$ & $0.633(4)$ & $0.125(4)$ & $-0.06(2)$ & 0.57 & 1.00 & $1.92(3)$ \\
\hline 2 & $\mathrm{O}_{2}(+)$ & 0.357 & 0.365 & 0.135 & 2.00 & $0.353(4)$ & $0.367(4)$ & $0.125(4)$ & $-0.06(2)$ & 0.57 & 1.00 & $1.92(3)$ \\
\hline 2 & $\mathrm{Mn}_{2}$ & 0.500 & 1.000 & 0.000 & 2.04 & $0.496(2)$ & $0.992(2)$ & $-0.003(2)$ & $-0.01(1)$ & 0.51 & 1.00 & $2.07(3)$ \\
\hline 2 & $\mathrm{Mn}_{2}$ & 0.000 & 0.000 & 0.000 & 2.04 & $-0.004(2)$ & $0.008(2)$ & $-0.003(2)$ & $-0.01(1)$ & 0.51 & 1.00 & $2.07(3)$ \\
\hline 2 & $\mathrm{C}_{2}$ & 0.250 & 0.500 & 0.000 & 3.96 & $0.247(4)$ & $0.500(4)$ & $-0.011(4)$ & $-0.06(2)$ & 0.48 & 1.00 & $3.96(3)$ \\
\hline 2 & $\mathrm{C}_{2}$ & 0.750 & 0.500 & 0.000 & 3.96 & $0.747(4)$ & $0.500(4)$ & $-0.011(4)$ & $-0.06(2)$ & 0.48 & 1.00 & $3.96(3)$ \\
\hline 2 & $\mathrm{O}_{2}$ & 0.250 & 0.770 & 0.000 & 2.00 & $0.247(4)$ & $0.770(4)$ & $-0.013(4)$ & $-0.08(2)$ & 0.57 & 1.00 & $2.00(3)$ \\
\hline 2 & $\mathrm{O}_{2}$ & 0.750 & 0.230 & 0.000 & 2.00 & $0.747(4)$ & $0.230(4)$ & $-0.013(4)$ & $-0.08(2)$ & 0.57 & 1.00 & $2.00(3)$ \\
\hline 2 & $\mathrm{O}_{2}(-)$ & 0.143 & 0.365 & -0.135 & 2.00 & $0.140(4)$ & $0.364(4)$ & $-0.146(4)$ & $-0.06(2)$ & 0.57 & 1.00 & $2.07(3)$ \\
\hline 2 & $\mathrm{O}_{2}(-)$ & 0.643 & 0.635 & -0.135 & 2.00 & $0.640(4)$ & $0.636(4)$ & $-0.146(4)$ & $-0.06(2)$ & 0.57 & 1.00 & $2.07(3)$ \\
\hline
\end{tabular}

Table S1 
Table S1. (A) Fractional $x-y-z$ coordinates and bond-valence sums ( $\Sigma \mathrm{BV}$ ) of atoms in the surface model for a bulk termination and $(B, C)$ fractional coordinates, Debye-Waller factors (DW), occupancies (Occ), and bond-valence sums of the optimized models without and with an oxygen overlayer. The cell parameters are $\left|\mathbf{a}_{\mathbf{s}}\right|=7.581 \AA,\left|\mathbf{b}_{\mathbf{s}}\right|=4.768 \AA,\left|\mathbf{c}_{\mathbf{s}}\right|=5.678 \AA$, and $\alpha=\beta$ $=\gamma=90^{\circ}$. The $\Delta z$ values (expressed in $\AA$ ) are the changes in the $z$-positioning of the layers with respect to the bulk termination. Subscript numbers indicate the layer where the atoms reside. Signs (+) and (-) correspond to higher and lower $z$-positions of oxygen compared to carbon in the same carbonate (see Figure 4). Estimated errors from the fit at the 95\% confidence level are given in parentheses. Values without reported errors were fixed in the fit or the errors are several magnitudes smaller than the calculated values. The error values are applied to the last digit of the calculated values (e.g., 0.707(4) = $0.707 \pm 0.004)$. 


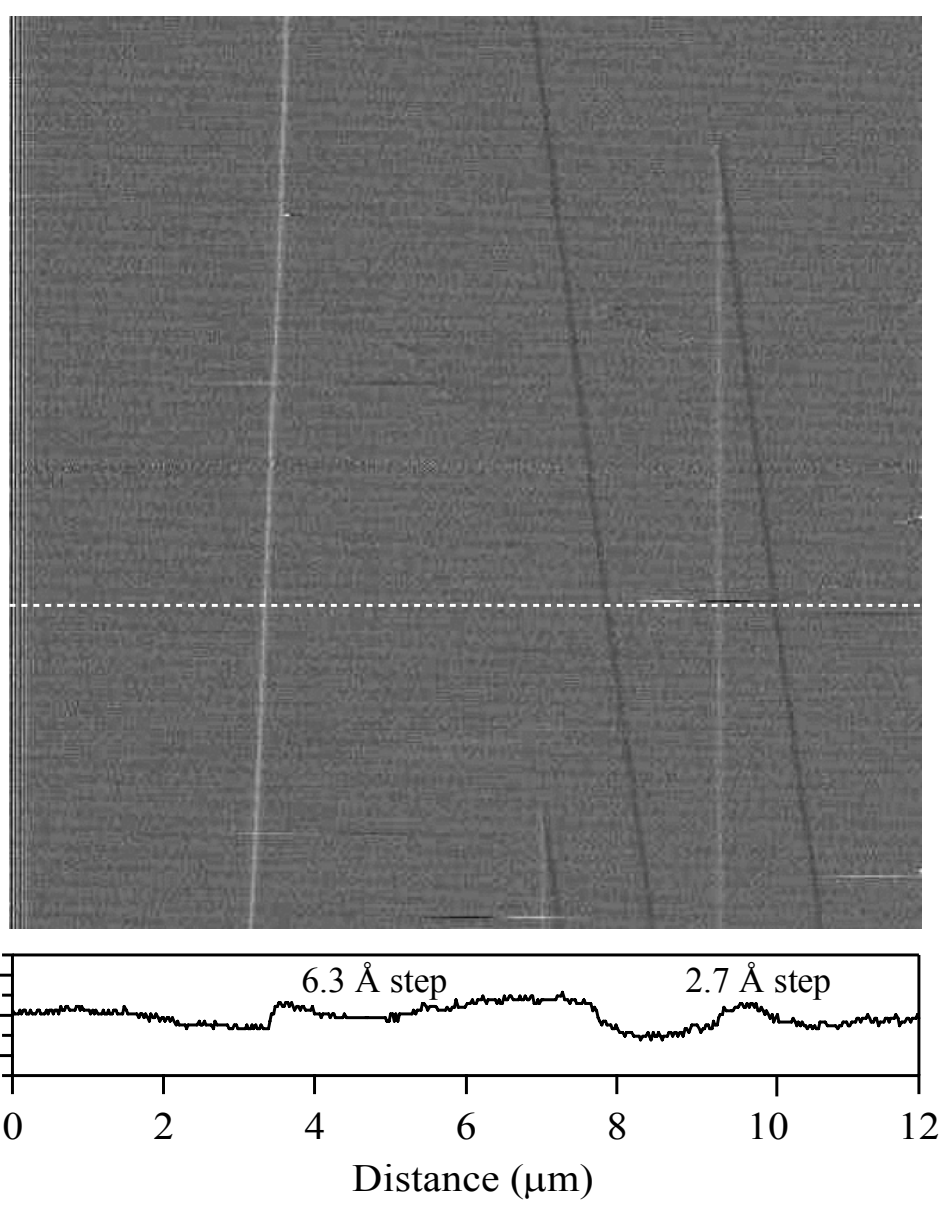

Figure S1. AFM micrograph of a freshly cleaved surface of $\mathrm{MnCO}_{3}$. The micrograph is shown in deflection mode $\left(12 \times 12 \mu^{2}\right)$ during exposure at $295 \mathrm{~K}$ to a solution having $\mathrm{pH}=6.4$. The height cross section corresponds to the white dotted line in the micrograph. Except for the presence of several steps, the surface terrace is smooth. There is low surface roughness on the individual terraces. The waviness of AFM image is the artifact from the instrument. From the least-squares CTR analysis, a root-mean-square roughness of $1.39 \pm$ $0.45 \AA$ is determined, which is consistent with the AFM observations of a large terrace morphology, with roughness of individual terraces on the order of a few angstroms (essentially at the noise floor of the instrument). The measurements are taken from the central areas of the test structures in order to avoid edge effects. 


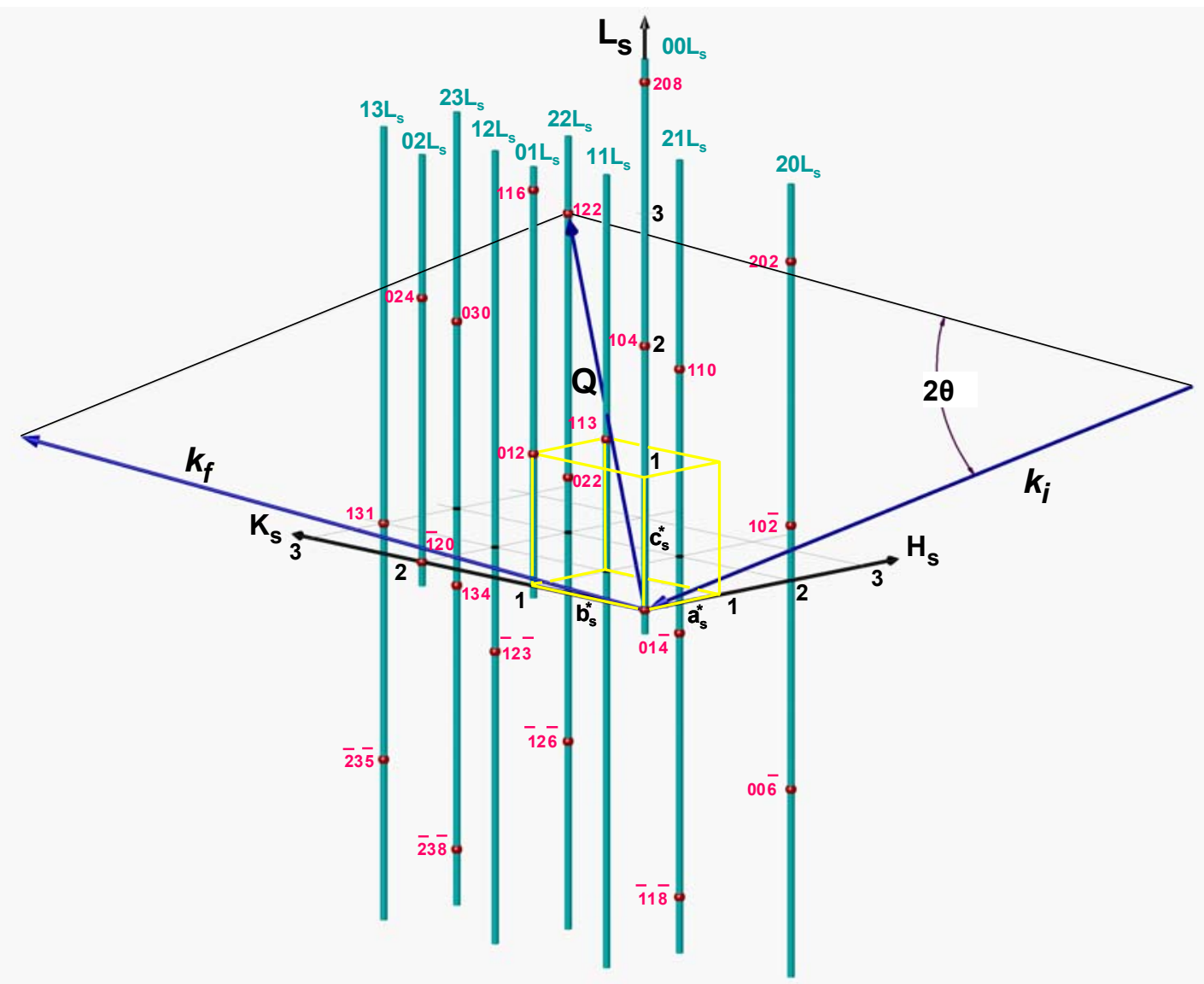

Figure S2. A section of reciprocal space plotted in the surface-indexed coordinate system. The values of $H_{s}, K_{s}$, and $L_{s}$ along the three perpendicular axes respectively correspond to the vectors $\mathbf{a}_{\mathrm{s}}^{*}, \mathbf{b}_{\mathrm{s}}$, and $\mathbf{c}_{\mathrm{s}}{ }_{\mathrm{s}}$ of the (10 $\left.\overline{1} 4\right)$-surface reciprocal lattice of $\mathrm{MnCO}_{3}$ (shown in yellow). Values are expressed as reciprocallattice units (r.l.u.). Light blue vertical lines (called rods) show sweeps in reciprocal space along the ten measured CTRs. A position along one of these lines corresponds to the $L_{s}$-axes of Figures 2 and 3. Red dots, which are labeled using bulk indexing, show the $H_{s}-K_{s}-L_{s}$ positions corresponding to the Bragg peaks of the bulk crystal. The shown wavevectors $\boldsymbol{k}_{\boldsymbol{i}}$ and $\boldsymbol{k}_{\boldsymbol{f}}$ of incident and scattered radiation illustrate the geometry satisfying the diffraction condition for the (122) Bragg peak (i.e., momentum transfer $\boldsymbol{Q}$ given by $\boldsymbol{k}_{\boldsymbol{i}}-\boldsymbol{k}_{\boldsymbol{f}}$ terminates at the (122) position). The CTRs are perpendicular to the $H_{s}-K_{s}$ plane. 


\section{Appendix S1. Surface indexing and the Bragg condition}

The periodicity of an infinite crystal can be fully represented by a complete set of reciprocal-lattice vectors $\boldsymbol{G}_{\boldsymbol{H} K \boldsymbol{L}}$. These vectors, which are normal to the atomic planes of the crystal, have lengths equal to the reciprocal of the interplanar spacing. The labels $H$, $K$, and $L$ correspond to the Miller indices of the atomic planes (1). The reciprocal-space representation of the three-dimensional periodicity of the $\mathrm{MnCO}_{3}$ crystal is shown in Figure S2 by the red dots, which correspond to the terminations of the set of $\boldsymbol{G}_{\boldsymbol{H} K L}$ vectors drawn from the origin.

Figure S2 also shows a surface-indexing coordinate system for the (10 $\overline{1} 4)$ termination of $\mathrm{MnCO}_{3}$ crystal structure. A unit cell is constructed with orthogonal axes $\mathbf{a}_{\mathbf{s}}-\mathbf{b}_{\mathbf{s}}-\mathbf{c}_{\mathbf{s}}$ such that the $\mathbf{a}_{\mathbf{s}}-$ and $\mathbf{b}_{\mathbf{s}}$-axes lie in-plane with the surface and the $\mathbf{c}_{\mathbf{s}}$ axis is normal to the surface. The corresponding axes of reciprocal space are denoted by $\mathbf{a}_{\mathrm{s}}{ }_{\mathrm{s}}-\mathbf{b}{ }_{\mathrm{s}}-\mathbf{c}_{\mathrm{s}}{ }_{\mathrm{s}}$ and for the case presented here have the relationship a ${ }_{\mathrm{s}}{ }_{\mathrm{s}}=2 \pi / \mathrm{a}_{\mathrm{s}}$ and so on. The axis $H_{s}-K_{s}-L_{s}$ are the indices of reciprocal lattice vectors, where $\mathbf{a}_{\mathrm{s}}^{*}, \mathbf{b}_{\mathrm{s}}^{*}$ and $\mathbf{c}_{\mathrm{s}}^{*}$ are the basis set defining the coordinate system.

The incident and the scattered x-ray beams are represented in Figure S2 by wavevectors, which are labeled $\boldsymbol{k}_{\boldsymbol{i}}$ and $\boldsymbol{k}_{\boldsymbol{f}}$, respectively. The wavevectors specify the beam direction and the reciprocal of the x-ray wavelength. For our elastic scattering experiment, the magnitudes of $\boldsymbol{k}_{\boldsymbol{i}}$ and $\boldsymbol{k}_{\boldsymbol{f}}$ are equal. For constructive interference of the incoming and the outgoing radiation, the momentum-transfer vector $\boldsymbol{Q}$, defined as $\boldsymbol{Q}=\boldsymbol{k}_{f}-\boldsymbol{k}_{i}=2 \pi\left(H_{s} \mathbf{a}_{s}^{*}+K_{s} \mathbf{b}_{s}^{*}+L_{s} \mathbf{c}_{s}^{*}\right)$, must coincide with one of the reciprocal-lattice vectors $\boldsymbol{G}_{\boldsymbol{H} \boldsymbol{}} \boldsymbol{L}$, thus defining the Bragg condition. As an example, Figure S2 shows the wavevectors $\boldsymbol{k}_{\boldsymbol{f}}$ and $\boldsymbol{k}_{\boldsymbol{i}}$ satisfying this condition for the (122) Bragg reflection. 


\section{Appendix S2. Basic theory for analysis by crystal-truncation rods}

To introduce the concept of scattering along crystal-truncation rods, we first recall that the classic explanation of x-ray scattering from a crystalline material describes scattering intensity as confined in reciprocal space to multiple, finite, and approximately spherically bounded regions. These regions are compact, contain most of the scattered xray intensity, and are centered at the reciprocal-lattice points (also known as Bragg points and denoted by the red dots in Figure S2). Among other factors, the exact shapes of these bounded regions depend on the quality, the shape, and the size of the physical crystal. Of particular interest to our presentation is that an inverse relation exists between the crystal size (i.e., three-dimensional truncation) and the size of the bounded region. This effect occurs because of the truncation of infinite periodicity of a crystal by its physical surfaces. In the case of three-dimensional truncation, therefore, finite-size broadening induces a significant expansion of the bounded region for sufficiently small crystals. For typical measurements, for which the crystal size is greater than a few microns, the broadening is negligible compared to the instrument resolution, and the scattering by the crystal effectively approaches the infinite-size limit.

Having considered the case of three-dimensional truncation, we can next consider the case of one-dimensional truncation. Notably, our study conditions correspond to one-dimensional truncation because a large $\mathrm{MnCO}_{3}$ crystal (ca. $3 \mathrm{~mm} \times 3 \mathrm{~mm}$ ) having an exposed $(10 \overline{1} 4)$ surface is irradiated by an x-ray beam having a cross section of $200 \mu \mathrm{m} \times 1000 \mu \mathrm{m}$.

Perturbations to the idealized, spherically bounded regions centered at the Bragg points occur when the model of an infinite crystal is replaced by one having one- 
dimensional truncation (i.e., a surface). The surface interrupts the periodicity and therefore complete destructive interference in one direction (i.e., the surface-normal direction). The surface truncation thereby causes systematic changes in the angular distribution of the scattered x-ray intensity. These changes map into reciprocal space as bounded tails extending from the spherically bounded regions that are parallel to the surface normal (2). The tails, which are narrow in the $H_{s}-K_{s}$ direction because of the nearly infinite extent of the crystal in the two non-truncated directions, join Bragg reflections along the $L_{s}$ direction. The resulting object in reciprocal space is called a crystal-truncation rod $(3,4)$ (e.g., a light blue vertical line in Figure S2).

The scattered x-ray intensity along the CTRs is the fundamental data employed in this study (see Figures 2 and 3). The intensity along CTRs is typically eight orders of magnitude lower than that occurring for bulk Bragg peaks because the scattering arises from just the approximately $10^{15}$ surface-region atoms located within the beam footprint. The high brilliance of synchrotron sources allows this much smaller amount of scattering to be investigated. The scattering intensity along CTRs is sensitive to the alternation of atomic positions in the near surface region compared to those in the bulk (5). Moreover, some rods are more selective than others to specific atoms and to specific vertical or lateral changes in positions. The ten CTRs corresponding to the data shown in Figures 2 and 3 are illustrated by the light blue lines in Figure S2.

In the surface-indexing system, rods are at integer $H_{s}$ and $K_{s}$ coordinates (light blue labels in Figure S2) and are continuous in the $L_{s}$ direction (6). The position along one CTR corresponds to a position along an $L_{s}$-axis of Figures 2 and 3. The $\left(00 L_{s}\right)$ rod is observed using specular scattering geometry with respect to the (10 $\overline{1} 4)$ surface, for 
which the incident and the scattered beams are at equal angles to the physical surface of the cleaved crystal. The other rods in Figure S2 are observed by employing nonspecular geometries, where the incident and the scattered beams define a plane that is not perpendicular to the physical surface.

The scattering intensity along a CTR is proportional to $\left|F_{T}\right|^{2}$, where $F_{T}$ is the calculated structure factor for a truncated crystal. The complex number $F_{T}$, which defines both intensity and phase, is calculated as follows ( 6 ):

$$
F_{T}=S \rho\left(F_{b} F_{C T R}+\sum_{j=1}^{m} f_{j} \exp \left(i \boldsymbol{Q} \cdot \mathbf{r}_{j}\right) \exp \left[-\frac{1}{2}\left(|\boldsymbol{Q}| u_{j}\right)^{2}\right]\right)
$$

where $S$ is an overall scale factor for the instrumentation, $\rho$ is the surface roughness factor $(2), F_{b}$ is the bulk structure factor, and $F_{\text {CTR }}=1 /\left[1-\exp \left(-i 2 \pi L_{s}\right)\right]$. The summation is over all atoms $m$ in the surface unit cell, where $f_{j}, \mathbf{r}_{j}$, and $u_{j}$ are the scattering factor, the position, and the isotropic vibrational amplitude (7), respectively, of surface atom $j$. The effect of anisotropic vibration is insignificant within measurement uncertainty and is therefore excluded in the calculations of the structure factor. The measured scattering intensity depends on the structure factor as well as beam intensity, sample-to-detector distance, and other factors (8).

\section{Literature Cited}

(1) Warren, B. E. X-ray Diffraction; Dover: New York, 1990.

(2) Robinson, I. K.; Tweet, D. J. Surface x-ray diffraction. Rep. Prog. Phys. 1992, 55, 599-651.

(3) Robinson, I. K. Crystal truncation rods and surface roughness. Phys. Rev. B 1986, 33, 3830-3836.

(4) Andrews, S. R.; Cowley, R. A. Scattering of x-rays from crystal-surfaces. J. Phys. C. Solid State 1985, 18, 6427-6439. 
(5) Trainor, T. P.; Chaka, A. M.; Eng, P. J.; Newville, M.; Waychunas, G. A.; Catalano, J. G.; Brown, G. E. Structure and reactivity of the hydrated hematite (0001) surface. Surf. Sci. 2004, 573, 204-224.

(6) Trainor, T. P.; Eng, P. J.; Robinson, I. K. Calculation of crystal truncation rod structure factors for arbitrary rational surface terminations. J. Appl. Crystallogr. 2002, 35, 696-701.

(7) Effenberger, H.; Mereiter, K.; Zemann, J. Crystal-structure refinements of magnesite, calcite, rhodochrosite, siderite, smithonite, and dolomite, with discussion of some aspects of the stereochemistry of calcite type carbonates. Z. Kristallogr. 1981, 156, 233-243.

(8) Vlieg, E. ROD: A program for surface x-ray crystallography. J. Appl. Crystallogr. 2000, 33, 401-405. 